\title{
ANÁLISE DO NÍVEL DE DIVULGAÇÃO DO RISCO OPERACIONAL SEGUNDO RECOMENDAÇÕES DO COMITÊ DA BASILÉIA: ESTUDO EM BANCOS DO PAÍS E DO EXTERIOR
}

\section{ANALYSIS OF OPERATIONAL RISK DISCLOSURE LEVEL ACCORDING TO RECOMMENDATIONS OF BASEL COMMITTEE: A STUDY IN BRAZILIAN AND FOREIGN BANKS}

\section{CARLOS ANDRÉ DE MELO ALVES}

Mestre em Administração pela Universidade Federal do Paraná (UFPR). Doutorando em Administração pela Faculdade de Economia, Administração e Contabilidade da Universidade de São Paulo (USP). Avenida Professor Luciano Gualberto, 908, Cidade Universitária - São Paulo - SP - CEP 05508-010 E-mail: camelodfpr@yahoo.com.br

ANA PAULA MUSSI SZABO CHEROBIM Doutora em Administração pela Universidade de São Paulo (USP). Professora adjunta III do Departamento de Administração Geral e Aplicada da Universidade Federal do Paraná (UFPR.) Rua Lothario Meissner, 632, $2^{\circ}$ andar, Jardim Botânico - Curitiba - PR - CEP 80210-170 E-mail: anapaulamussi@ufpr.br 


\section{RESUMO}

Este estudo buscou verificar os níveis de divulgação do risco operacional de 24 bancos do país e de 9 do exterior, segundo recomendações do Comitê da Basiléia, em 3I de dezembro de 2003 e 3I de dezembro de 2004. Realizaram-se revisão teórica e pesquisa documental, com o propósito de analisar o conteúdo de relatórios anuais e outros instrumentos de divulgação. A amostra baseou-se no relatório "50 maiores bancos" do Banco Central do Brasil. A análise empregou 9 categorias e I8 subcategorias baseadas em recomendações do Comitê, na revisão teórica e em pesquisa exploratória em que se coletou opinião de profissionais relativa às categorias e subcategorias. Aplicaram-se testes não paramétricos. Constatou-se que os níveis de divulgação de bancos do país e do exterior em 2003 foram, respectivamente, I7,I3\% e 77,78\%, e em 2004, 24,31\% e 8I,48\%. Os testes indicaram que os bancos do exterior divulgaram mais subcategorias e que a quantidade de subcategorias divulgadas pelos dois grupos de bancos aumentou em 2004 .

\section{PALAVRAS-CHAVE}

Risco operacional; Divulgação do risco operacional; Recomendações do Comitê da Basiléia; Instituições financeiras; Testes não paramétricos.

\section{ABSTRACT}

This paper examines the operational risk disclosure levels of 24 brazilian banks and 9 foreign banks based on the Basel Committee recommendations. The information is taken from 2003 and 2004 annual reports and other disclosure instruments. The sample is based on Central Bank of Brazil's Report "50 maiores bancos". It was used an analysis framework of 9 categories and 18 subcategories, based on the Pillar 3 of Basel II, in the "Sound Practices for the Management and Supervision of Operational Risk", in the theoretical review and in a research in which were considered professionals opinions about the categories and subca- 
tegories. Non-parametric tests were used. The results showed that the disclosure levels for brazilian and foreign banks, respectively, were of $17.13 \%$ and $77.78 \%$ in 2003 , and $24.31 \%$ and $81.48 \%$ in 2004 . The non-parametric tests showed that foreign banks disclosed more than brazilian banks, and both increased their disclosure levels in 2004 .

\section{KEYWORDS}

Operational risk; Operational risk disclosure; Recommendations of the Basel Committee; Financial institutions; Non-parametric tests.

\section{INTRODUÇÃO}

Nos últimos anos, os avanços tecnológicos, como as negociações bancárias via internet, a sofisticação de produtos e serviços oferecidos pelos bancos, a ocorrência de escândalos financeiros relacionados a fraudes, entre outros exemplos, contribuíram para que instituições financeiras e autoridades reguladoras passassem a dar mais atenção ao risco operacional como um tipo de risco merecedor de tratamento corporativo.

O risco operacional é objeto de interesse de bancos e de supervisores bancários de vários países. O Comitê de Supervisão Bancária da Basiléia ${ }^{\mathrm{T}}$ é uma entidade com respaldo internacional que estimula o tratamento do risco operacional, inclusive a divulgação ${ }^{2}$ de informações relacionadas a esse tipo de risco.

A divulgação de informações sobre gestão de riscos, inclusive a gestão do risco operacional, contribui para a transparência dos atos de gestão de instituições financeiras perante investidores e para a disciplina do mercado. Duas recomendações elaboradas pelo Comitê da Basiléia podem ser associadas à divulgação do risco operacional: o acordo "Basiléia II", publicado em junho de 2004 e atualizado em novembro de 2005 (BASEL COMMITTEE..., 2005), e o documento "Boas práticas para o gerenciamento e supervisão do risco operacional" (BASEL COMMITTEE..., 2003b).

O Comitê de Supervisão Bancária da Basiléia (também chamado "Comitê da Basiléia" ou simplesmente "Comitê"), de acordo com BCBS (2005, p. I), "é um comitê de autoridades de supervisão bancária que foi estabelecido pelos presidentes dos bancos centrais dos países do Grupo dos Dez em I975. É constituído por representantes de autoridades de supervisão bancária e de bancos centrais dos seguintes países: Bélgica, Canadá, França, Alemanha, Itália, Japão, Luxemburgo, Holanda, Espanha, Suécia, Suíça, Reino Unido e Estados Unidos".

2 Neste estudo, os termos divulgação, evidenciação e disclosure são considerados sinônimos. 
No Brasil, a divulgação do risco operacional foi voluntária no período a que se refere este estudo. No entanto, a não-obrigatoriedade da divulgação desse risco não indica necessariamente que acionistas e stakeholders prescindam desse tipo de informação. Ainda que as técnicas de avaliação de riscos operacionais não estejam totalmente desenvolvidas, segundo o Basel Committee on Banking Supervision (BCBS) (2003b, p. I5),

[...] um banco deve divulgar sua estrutura de gestão do risco operacional de tal maneira que permita que investidores e contrapartes possam determinar se um banco efetivamente identifica, avalia, monitora e controla/mitiga risco operacional.

Dessa forma, o objetivo deste trabalho foi verificar os níveis de divulgação do risco operacional de 24 bancos do país e de 9 do exterior, segundo recomendações do Comitê da Basiléia, nas datas-base 3I de dezembro de 2003 e 3I de dezembro de 2004 .

Para tal fim, realizaram-se revisão teórica e pesquisa documental, com análise de conteúdo em relatórios anuais e outros instrumentos de divulgação. A análise empregou nove categorias e I8 subcategorias, baseadas em recomendações do Comitê, na revisão teórica e em pesquisa exploratória na qual se coletou opinião de profissionais a respeito das categorias e subcategorias. Foram aplicados testes não paramétricos para dar significância estatística às análises dos dados.

O interesse em analisar a divulgação do risco operacional também reside no fato de que o tema deste artigo é atual e desperta atenção tanto do público acadêmico quanto dos profissionais ligados ao mercado financeiro, tendo em vista as exigências para implantação das diretrizes baseadas no "Basiléia II", relativas ao risco operacional. No país, tais diretrizes estão presentes nos Comunicados n ${ }^{\circ}$ I2.746 e I6.I37, do Banco Central do Brasil, e na Resolução $\mathrm{n}^{\circ} 3.380$, do Conselho Monetário Nacional, a qual dispõe a respeito da implementação da estrutura de gerenciamento do risco operacional em instituições financeiras.

Além desta introdução, o estudo apresenta revisão teórica contemplando o risco operacional, recomendações do Comitê da Basiléia relacionadas à divulgação desse risco e pesquisas anteriores a respeito da divulgação do risco operacional em bancos. Em seguida, apresentam-se a metodologia empregada para atingir os objetivos do estudo, os resultados obtidos, a conclusão e finalmente as referências bibliográficas. 


\section{REVISÃO TEÓRICA}

Esta revisão apresenta o risco operacional, as recomendações do Comitê da Basiléia relacionadas à divulgação desse risco e as pesquisas empíricas a respeito da divulgação do risco operacional que serviram de referência para este trabalho.

\subsection{RISCO OPERACIONAL}

Risco operacional não é um tema pacífico na literatura. Segundo Crouhy, Galai e Mark (200I, p. 475) "[...] é difícil fazer uma clara distinção entre risco operacional e as incertezas 'normais' enfrentadas pelas organizações em suas operações diárias". Apesar disso, autores e autoridades reguladoras buscaram conceituar esse risco.

De acordo com o Comitê da Basiléia (BCBS, 2005, p. I40),

risco operacional é definido como o risco de perdas resultantes de processos internos falhos ou inadequados, pessoas e sistemas, ou eventos externos. A definição inclui risco legal, mas exclui risco estratégico e reputacional.

O referido Comitê divulgou uma classificação por sete tipos de evento para o risco operacional: fraudes internas; fraudes externas; práticas empregatícias e segurança no ambiente de trabalho; clientes, produtos e práticas de negócios; danos a ativos físicos; interrupção dos negócios e falhas de sistemas; execução, entrega e gestão de processos (BCBS, 2003b).

Segundo o BCBS (2003b, p. 2), a gestão do risco operacional pode ser entendida como "[...] identificação, avaliação, monitoramento e controle/mitigação do risco". O tratamento do risco operacional pode ser compreendido em perspectiva qualitativa, quantitativa ou ambas.

Padrões qualitativos podem referir-se a diretrizes de controles internos, diretrizes de qualidade para processos e recursos, e diretrizes setoriais de boas práticas operacionais. A perspectiva quantitativa pode associar-se à utilização de modelos para mensuração do risco operacional, com o uso de técnicas como value at risk, por exemplo.

Por fim, o risco operacional possui regulamentação no Brasil contemplada na Resolução do Conselho Monetário Nacional n 3.380, de 29 de junho de 2006 . Essa resolução foi mencionada na introdução deste estudo e dispõe a respeito da implementação da estrutura de gerenciamento do risco operacional aplicável às instituições financeiras. Entre os aspectos nela inseridos, encontram-se a obriga- 
toriedade de uma estrutura mínima para tratar o risco operacional da instituição financeira, contendo a atribuição de um diretor responsável por tal estrutura, a segregação entre as atividades de gestão de risco operacional e de auditoria interna, e a obrigatoriedade da evidenciação de informações a respeito da estrutura de risco operacional em relatório de acesso público.

\subsection{RECOMENDAÇÕES DO COMITÊ DA BASILÉIA RELATIVAS À DIVULGAÇÃO DO RISCO OPERACIONAL}

Abordam-se aqui duas recomendações do Comitê da Basiléia que podem ser associadas à divulgação do risco operacional: "Basiléia II" e "Boas práticas para o gerenciamento e supervisão do risco operacional”.

O Comitê da Basiléia apresentou o acordo "Convergência internacional de mensuração e padrões de capital: uma estrutura revisada", conhecido por "Basiléia II" (BCBS, 2005). Esse acordo inova, entre outros pontos, ao contemplar o risco operacional.

O “Basiléia II" subdivide-se em três pilares:

- Pilar 1: relaciona-se com requerimentos mínimos de capital regulamentar para os riscos aos quais as instituições financeiras estão expostas³.

- $\quad$ Pilar 2: relaciona-se com o processo de revisão da supervisão.

- Pilar 3: relaciona-se com a transparência e disciplina do mercado (BCBS, 2005).

O pilar 3 contém requisitos de divulgação de informações financeiras, os quais podem ajudar na verificação do nível de divulgação de bancos. Neste trabalho, "nível de divulgação" "pressupõe um volume mínimo de divulgação compatível com o objetivo negativo de evitar que as demonstrações sejam enganadoras" (HENDRIKSEN; BREDA, I999, p. 5I5). Vale dizer que esse pilar contempla requisitos de divulgação do risco operacional.

No Brasil, o Banco Central (2007a) manifestou-se quanto à implementação das diretrizes do "Basiléia II" no país por meio do Comunicado n ${ }^{\circ}$ 2.746, de 9 de dezembro de 2004. O comunicado apresenta cronograma de implantação que

\footnotetext{
Apesar de não ser o objetivo deste estudo, deve-se mencionar que o pilar I contempla requerimentos de capital regulamentar para o risco operacional, destacando-se as seguintes abordagens para mensuração, segundo a ordem de sofisticação e sensibilidade ao risco: abordagem de indicador básico (basic indicator approach - BIA), abordagem padronizada (stantardized approach - SA), abordagem padronizada alternativa (alternative standardized approach - ASA) e abordagem de mensuração avançada (advanced measurement approach - AMA). A AMA preconiza a utilização de modelos internos para mensurar o risco operacional, considerando uma base de dados de perdas operacionais (BCBS, 2005).
} 
se estende até 20II. Posteriormente, o Comunicado ${ }^{\circ}$ I6.I37, de 27 de setembro de 2007 , estendeu a implantação até 2012 e salientou que permanecem válidas as diretrizes para implementação dispostas no Comunicado ${ }^{\circ}$ I2.746, entre elas a previsão para requerer parcela de capital dos bancos para cobrir riscos operacionais e a aplicação do pilar 3 a todas instituições do sistema financeiro nacional (SFN).

A divulgação do risco operacional também é mencionada no documento recomendado pelo Comitê da Basiléia denominado "Boas práticas para o gerenciamento e supervisão do risco Operacional”, também chamado neste estudo de "Boas práticas" (BCBS, 2003b).

O "Boas práticas" contém

[...] um conjunto de princípios que fornecem uma estrutura para o efetivo gerenciamento e para a supervisão do risco operacional que poderão ser utilizados por bancos e autoridades supervisoras na avaliação de políticas e práticas de gerenciamento de risco operacional (BCBS, 2003b, p. I).

O Quadro I enumera as práticas e os princípios recomendados pelo Comitê da Basiléia contidos no documento "Boas práticas".

\section{QUADRO I}

PRÁTICAS E PRINCÍPIOS RECOMENDADOS PELO COMITÊ DA BASILÉIA

\begin{tabular}{lc}
\hline PRÁTICAS & PRINCíPIOS \\
\hline Desenvolvimento de um ambiente apropriado ao gerenciamento de risco & De 1 a 3 \\
\hline Identificação, avaliação, monitoramento e controle/mitigação do risco & De 4 a 7 \\
\hline Papel dos supervisores & 8 e 9 \\
\hline Papel da divulgação & 10 \\
\hline
\end{tabular}

Fonte: BCBS (2003b).

De acordo com Eagovil (2003), os princípios contidos no documento "Boas práticas" direcionado a bancos (de I a 7 e Io) guardam intersecção com práticas de governança corporativa.

Um dos elementos destacado no documento "Boas práticas" refere-se às ferramentas para identificar e avaliar o risco operacional. Mesmo que não sejam literalmente ferramentas, é relevante destacar os mecanismos de incentivo à 
coleta de perdas operacionais. Por exemplo, conforme lembra Ribeiro (2003), a adoção de programa de remuneração variável foi fator diferencial na implantação de etapa do projeto de gestão do risco operacional em agências de instituição financeira de grande porte no país.

Finalmente, deve-se destacar que o papel da divulgação do risco operacional é enfatizado no princípio ıo, tal como mostra o Quadro I. De acordo com o Comitê da Basiléia, os bancos devem promover a divulgação de informações relacionadas ao risco operacional, ainda que as técnicas de avaliação dos riscos operacionais não estejam totalmente desenvolvidas (BCBS, 2003b).

\subsection{ESTUDOS EMPÍRICOS RELACIONADOS À DIVULGAÇÃO DO RISCO OPERACIONAL EM BANCOS}

Neste tópico, apresentam-se estudos empíricos relacionados à divulgação do risco operacional em bancos. A divulgação do risco foi abordada em Helbok e Wagner (2004) e Carvalho, Trapp e Chan (2004). Mesmo não sendo o foco dos estudos, a divulgação do risco operacional também foi contemplada nas pesquisas empíricas realizadas pelo Comitê da Basiléia em I999 (BCBS, 200I), 2000 (BCBS, 2002) e 200I (BCBS, 2003a), e em Xavier (2003). Finalmente, em Barroso, Lustosa e Moraes (2004) e Sundmacher (2006) são apresentadas contribuições para a revisão teórica a respeito desse tema.

Helbok e Wagner (2004) apresentaram estudo internacional a respeito da divulgação corporativa do risco operacional em bancos comerciais, com base nos anos de I998 a 200I. No período do levantamento, a divulgação desse risco foi voluntária. Utilizou-se um índice para pesquisar o nível de divulgação em relatórios anuais de bancos. Esses autores concluíram que, de I998 a 200I, houve um aumento substancial tanto na extensão quanto na qualidade da divulgação financeira corporativa de bancos em risco operacional. Sugere-se que preocupações com lucratividade podem levar instituições financeiras a terem um nível de divulgação do risco operacional mais alto, com a finalidade de garantir ao mercado que riscos operacionais estão sendo bem administrados.

Em Carvalho, Trapp e Chan (2004), analisou-se o estágio da divulgação do risco operacional das instituições financeiras que atuam no Brasil, comparando-as com as que atuam na Europa e nos Estados Unidos. Foram estudados os demonstrativos financeiros correspondentes a 2002 e mensurou-se o nível de divulgação do risco operacional com base em parâmetros básicos do pilar 3 do "Basiléia II". Foi utilizado o teste não paramétrico qui-quadrado para testar a hipótese de igualdade de proporções entre as amostras de populações distintas. As amostras totalizaram II5 entidades que atuam no Brasil, 87 nos Estados Uni- 
dos e 65 na Europa. Com base no teste aplicado, não se constataram evidências que levassem à rejeição da hipótese nula de igualdade de proporções. Além disso, de acordo com Carvalho, Trapp e Chan (2004, p. 270)

verificou-se que no Brasil, na Europa e nos Estados Unidos, somente cerca de $7 \%$, I7\% e $7 \%$ das respectivas amostras apresentaram alguma iniciativa de evidenciação sobre esse assunto nas demonstrações contábeis.

As pesquisas empíricas realizadas pelo Comitê da Basiléia em I999 (BCBS, 200I), 2000 (BCBS, 2002) e 200I (BCBS, 2003a) versaram sobre a divulgação feita por bancos. Apesar de o risco operacional não ter recebido o mesmo tratamento dado ao risco de crédito e de mercado em termos de quantidade de questões nas pesquisas, de acordo com BCBS (2003a, p. 29),

[...] 91\% dos bancos forneceram informação sobre os principais tipos de risco operacional e identificaram e discutiram assuntos específicos considerados significantes nesse tópico (63\% em 1999). Oitenta e um por cento dos bancos também evidenciaram contingências legais e discutiram possíveis obrigações $(53 \%$ em 1999).

Um dos objetivos buscados em Xavier (2003, p. 5) foi comparar o grau de transparência das demonstrações contábeis publicadas pelos principais bancos brasileiros com os critérios de divulgação que poderão ser estabelecidos pelo "Basiléia II". Estudaram-se os relatórios anuais e demonstrativos financeiros dos dez principais conglomerados financeiros entre 200I e 2002. As categorias analisadas foram: escopo de aplicação, capital, exposição a risco e adequação de capital. Concluiu-se que a divulgação foi incipiente no período. Segundo Xavier (2003, p. 77),

tal conclusão, entre todas as observações feitas, está apoiada no fato de os bancos pesquisados pelo Comitê terem divulgado $56 \%$, $58 \%$ e $63 \%$ dos itens, nos anos de I999, 2000 e 200 , respectivamente, enquanto que os bancos objeto desta pesquisa divulgaram $25 \%$ e $26 \%$, nos anos de 200 I e 2002 .

No que se refere ao risco operacional, não incluindo o risco legal, não foram encontrados indícios de divulgação nas demonstrações pesquisadas no período. Quanto às contingências legais, a divulgação foi encontrada em $50 \%$ dos bancos em 2001, e em 60\% dos bancos em 2002. 
O objetivo em Barroso, Lustosa e Moraes (2004) foi avaliar o nível de evidenciação do risco operacional dos cinco maiores bancos do Brasil à luz do pilar 3 do "Basiléia II". Analisaram-se relatórios anuais de 2000 a 2003, e chegouse à conclusão de que, no período analisado, em média $25 \%$ das sugestões do Comitê foram divulgadas. Esse percentual praticamente se manteve constante ao longo dos anos do estudo. É mencionado no referido estudo que a divulgação não necessariamente reflete o estágio de evolução da gestão do risco operacional de um banco.

Finalmente, o objetivo de Sundmacher (2006) foi examinar a natureza da divulgação do risco operacional em bancos internacionais, a consistência dessa divulgação e sua utilidade. A análise baseou-se nos relatórios anuais de 2004 e de 2005 de 57 instituições financeiras internacionais e restringiu-se aos requerimentos presentes no pilar 3 relacionados ao risco operacional. Os resultados apontaram que a quantidade e a qualidade da divulgação variaram entre as instituições analisadas, e as informações foram predominantemente descritivas.

\section{METODOLOGIA}

Este tópico descreve a população e a amostra, a coleta e o tratamento dos dados, a elaboração do quadro usado para analisar o nível de divulgação do risco operacional, e, por fim, apresenta os testes não paramétricos utilizados nas análises dos resultados.

\subsection{POPULAÇÃO E AMOSTRA DE BANCOS}

A população de bancos do país deste estudo é constituída pelas instituições financeiras pertencentes ao Consolidado Bancário I presentes no relatório "50 maiores bancos e o consolidado do sistema financeiro nacional", data-base dezembro de 2004. Tais instituições são ordenadas por volume de ativos totais, deduzido da intermediação (títulos - posição financiada).

A amostra é não probabilística por julgamento. Foram selecionadas as entidades em ordem decrescente de volume de ativos totais, até que o acumulado ultrapassasse $95 \%$ do somatório dos ativos dos cinquenta maiores bancos. Justifica-se o percentual por ser significativo para a população, em termos de ativos totais. Das 25 entidades encontradas, foi retirada uma entidade que estava sob intervenção em 2004, chegando-se às 24 instituições descritas no Quadro 2.

Quanto ao cenário financeiro que se apresentou para os bancos do Quadro 2 no período de 2003 a 2004, destacamos que os eventos ocorridos no transcurso 
de 2004 não produziram efeitos capazes de impor profundas alterações na estrutura do sistema financeiro. Destaca-se no período a ampliação das alternativas de concessão de crédito no sistema financeiro em fins de 2003 , quando foi regulamentado o crédito consignado (BANCO CENTRAL DO BRASIL, 2007c).

\section{QUADRO 2}

\begin{tabular}{cllll} 
& \multicolumn{2}{c}{$\begin{array}{c}\text { AMOSTRA DE BANCOS DO PAÍS } \\
\text { (DATA-BASE: DEZEMBRO DE 2004) }\end{array}$} \\
\hline POSTO & INSTITUIÇÕES & POSTO & INSTITUIÇÕES \\
\hline 1 & BB & 13 & BANKBOSTON \\
\hline 2 & CEF & 14 & BNB \\
\hline 3 & BRADESCO & 15 & BANRISUL \\
\hline 4 & ITAÚ & 16 & CREDIT SUISSE \\
\hline 5 & UNIBANCO & 17 & ALFA \\
\hline 6 & SANTANDER BANESPA & 18 & JP MORGAN CHASE \\
\hline 7 & ABN AMRO & 19 & PACTUAL \\
\hline 8 & SAFRA & 20 & BNP PARIBAS \\
\hline 9 & HSBC & 21 & BBM \\
\hline 10 & NOSSA CAIXA & 22 & RURAL \\
\hline 11 & VOTORANTIM & 23 & DEUTSCHE \\
\hline 12 & CITIBANK & 24 & BIC \\
\hline
\end{tabular}

Fonte: Elaborado pelos autores com base no Banco Central do Brasil (2007c).

Além dos instrumentos divulgados pelos bancos do país, analisaram-se também os relatórios anuais de 2003 e 2004 de nove conglomerados financeiros do exterior. O critério para escolher tais conglomerados foi o fato de serem as matrizes, na data-base de dezembro de 2004 , dos nove bancos do país com controle estrangeiro que fazem parte da amostra de bancos nacionais deste estudo. A análise dos relatórios das matrizes de bancos com controle estrangeiro serviu como referência adicional para a análise da divulgação do risco operacional feita para bancos do país. 
O Quadro 3 apresenta a relação das matrizes de bancos com controle estrangeiro. Com relação a esse quadro, o Grupo Santander inclui o Banco Santander Central Hispano.

\section{QUADRO 3}

RELAÇÃO DAS MATRIZES DE BANCOS DO PAÍS COM CONTROLE ESTRANGEIRO (DATA-BASE: DEZEMBRO DE 2004)

\begin{tabular}{ll}
\hline BANCO DO PAÍS COM CONTROLE ESTRANGEIRO & MATRIZ (BANCO DO EXTERIOR) \\
\hline SANTANDER BANESPA & GRUPO SANTANDER \\
\hline ABN AMRO & ABN AMRO HOLDING N.V. \\
\hline HSBC & HSBC HOLDINGS PLC \\
\hline CITIBANK & CITIGROUP \\
\hline BANKBOSTON & BANK OF AMERICA CORPORATION \\
\hline CREDIT SUISSE & CREDIT SUISSE GROUP \\
\hline JP MORGAN CHASE & JP MORGAN CHASE \& CO. \\
\hline BNP PARIBAS & BNP PARIBAS \\
\hline DEUTSCHE & DEUTSCHE BANK AG \\
\hline
\end{tabular}

Fonte: Elaborado pelos autores com base em dados secundários da pesquisa.

Os bancos do Quadro 3 são sediados nos Estados Unidos e na Europa. Quanto ao cenário financeiro que se apresentou para esses bancos no período (2003 e 2004), destacamos que nos Estados Unidos o número de instituições com problemas diminuiu em 2004 em relação a 2003, e na Europa os bancos europeus, de forma geral, mantiveram níveis de solidez alcançados nos anos anteriores, logrando boa recuperação desde 2003 (BANCO CENTRAL DO BRASIL, 2008a).

Para as análises que seguem, todos os 24 bancos do Quadro 2 serão considerados bancos do país, e as matrizes de bancos com controle estrangeiro contidas na coluna à direita do Quadro 3 serão consideradas bancos do exterior 4 .

4 Em essência, o banco do país com controle estrangeiro faz parte do patrimônio da matriz. No entanto, com base no Comunicado $\mathrm{n}^{\circ}$ I2.746 do Banco Central, os bancos autorizados a funcionar no país não serão diferenciados entre si quanto à origem do capital no que se refere ao tratamento que será dado com base no "Basiléia II". Portanto, para os efeitos deste estudo, o banco autorizado a funcionar no país com controle estrangeiro pertence ao grupo de bancos do país, e sua matriz, ao grupo de bancos do exterior. Tal separação estende-se aos instrumentos de divulgação veiculados pelos bancos do país e do exterior. 
Todos os bancos divulgaram informações tanto em 2003 quanto em 2004. A escolha dessas datas foi justificada, pois a versão do "Basiléia II" e o Comunicado ${ }^{\circ}$ I2.746 do Banco Central do Brasil foram publicados em 2004, ou seja, analisaram-se informações publicadas pelos bancos antes e depois de terem sido divulgadas tais diretrizes, o que motivou verificar se houve mudança no nível de divulgação do risco operacional entre os dois períodos.

Para atingir os objetivos deste estudo, não foi necessário indicar o nome dos bancos nas análises. Bastou apresentar a quantidade de subcategorias divulgadas, substituindo o nome de cada entidade por um código que será uniforme no decorrer da narrativa e que não corresponde ao posto do banco em termos de ativos totais. Bancos do exterior foram tratados de forma similar aos bancos do país. Assim, a identidade dos bancos foi preservada.

\subsection{COLETA E TRATAMENTO DOS DADOS}

A coleta das fontes usadas na pesquisa documental foi feita nos sítios eletrônicos dos próprios bancos, da Comissão de Valores Mobiliários (CVM) e da Bolsa de Valores de São Paulo (Bovespa). Para quatro bancos, os instrumentos de divulgação foram coletados no sítio eletrônico do Diário Oficial do Estado de São Paulo, e, para um banco, os instrumentos de divulgação de 2003 foram coletados em jornal de grande circulação, sendo solicitado ao setor de atendimento do referido jornal o envio do exemplar pelo correio. Para bancos do exterior, os relatórios foram coletados nos respectivos sítios eletrônicos.

Utilizaram-se relatórios anuais e subsidiariamente outros instrumentos de divulgação, como demonstrações contábeis, notas explicativas, quadros suplementares às demonstrações contábeis, relatórios de administração, parecer dos auditores independentes e, para o período de 2004, os resumos do relatório do comitê de auditoria, quando disponíveis. Para bancos do exterior, foram usados exclusivamente relatórios anuais.

Para o tratamento dos dados, utilizou-se a análise de conteúdo, conceituada por Bardin (I977, p. 42) como

um conjunto de técnicas de análise das comunicações visando obter, por procedimentos sistemáticos e objetivos de descrição do conteúdo das mensagens, indicadores (quantitativos ou não) que permitam a inferência de conhecimentos relativos às condições de produção/recepção (variáveis inferidas) destas mensagens.

A análise de conteúdo adotada foi categorial temática. De acordo com Bardin (I977, p. I53), "funciona por operações de desmembramento do texto em unida- 
des, em categorias segundo reagrupamentos analógicos". Além disso, a análise foi qualitativa, e a regra de enumeração consistiu na presença ou ausência de termos.

A organização da análise apresentou três etapas, conforme Bardin (I977): pré-análise, exploração do material e tratamento dos dados, da inferência e da interpretação.

Na pré-análise, foram selecionados os documentos a serem examinados, e definiram-se as categorias e subcategorias utilizadas na análise de conteúdo. Durante essa etapa, realizou-se pesquisa exploratória, por meio de questionário, em que foi obtida a opinião de dez profissionais a respeito das categorias e subcategorias de análise elaboradas previamente com base na revisão teórica. $\mathrm{O}$ critério para escolha de tais profissionais foi terem publicado trabalhos a respeito do risco operacional ou atuarem na gestão de risco de bancos autorizados a funcionar no país. A pesquisa sobre as categorias e subcategorias baseou-se em esforço similar ao descrito em Shigvi e Desai (I97I), no qual se buscou a opinião de profissionais durante a elaboração de indicadores de análise utilizados em estudo de disclosure de informações financeiras. Essa pesquisa exploratória subsidiou a operacionalização das categorias que foram usadas na análise de conteúdo e que estão presentes no Quadro 4.

$\mathrm{Na}$ exploração do material, para cada banco foram examinados os instrumentos de divulgação e buscou-se verificar a presença ou ausência das I8 subcategorias distribuídas em nove categorias, como consta no Quadro 4.

No tratamento de resultados, inferência e interpretação, as análises foram registradas em tabela e apresentadas por subcategoria, como descrito no item 4.I, e apresentou-se o resultado para o grupo de bancos do país e exterior, tal como mostra o item 4.2 deste estudo.

\subsection{ELABORAÇÃO DO QUADRO DE ANÁLISE}

As categorias e subcategorias utilizadas na pesquisa estão descritas no Quadro 4, o qual apresenta 9 categorias e I8 subcategorias. As seis primeiras categorias associam-se a subcategorias predominantemente qualitativas, a sétima e oitava categorias associam-se a subcategorias quantitativas, e a nona categoria é predominantemente qualitativa. 


\section{QUADRO 4}

\section{QUADRO DE ANÁLISE USADO NO ESTUDO DO NÍVEL DE DIVULGACÃO DO RISCO OPERACIONAL}

\begin{tabular}{|c|c|}
\hline CATEGORIAS & SUBCATEGORIAS \\
\hline \multirow{3}{*}{$\begin{array}{l}\text { 1. Estratégias e } \\
\text { políticas }\end{array}$} & Descrição dos objetivos relacionados à gestão de riscos \\
\hline & Menção à integração de riscos \\
\hline & Conceito de risco operacional utilizado \\
\hline \multirow{4}{*}{$\begin{array}{l}\text { 2. Estrutura e } \\
\text { organização da } \\
\text { função }\end{array}$} & Envolvimento da alta administração com risco operacional \\
\hline & Estrutura organizacional responsável pelo risco operacional \\
\hline & Segregação entre gestão de riscos e área de negócios do banco \\
\hline & Segregação entre auditoria interna e gestão de riscos \\
\hline \multirow{2}{*}{$\begin{array}{l}\text { 3. Sistema de } \\
\text { reporte interno }\end{array}$} & Menção ao reporte da área de negócios para o gestor de risco \\
\hline & Menção ao reporte do gestor de risco para alta administração \\
\hline \multirow{2}{*}{$\begin{array}{l}\text { 4. Ferramentas para } \\
\text { identificação e } \\
\text { avaliação do risco }\end{array}$} & Menção a ferramentas para identificar e avaliar o risco operacional \\
\hline & Mecanismos de incentivo à coleta de perdas operacionais \\
\hline \multirow{3}{*}{$\begin{array}{l}\text { 5. Técnicas para } \\
\text { diminuição do } \\
\text { risco }\end{array}$} & Menção a instrumentos para transferência do risco \\
\hline & Menção a planos de contingência e/ou de continuidade de negócios \\
\hline & $\begin{array}{l}\text { Menção a meios para conscientização do corpo funcional quanto ao } \\
\text { risco }\end{array}$ \\
\hline $\begin{array}{l}\text { 6. Abordagem para } \\
\text { avaliação de } \\
\text { capital }\end{array}$ & $\begin{array}{l}\text { Menção à abordagem para avaliação de capital do risco para qual o } \\
\text { banco pretende se qualificar }\end{array}$ \\
\hline 7. Exposição ao risco & Exposição ao risco operacional (valores totais ou por linhas de negócios \\
\hline $\begin{array}{l}\text { 8. Encargo } \\
\text { de capital } \\
\text { regulamentar }\end{array}$ & $\begin{array}{l}\text { Encargo de capital regulamentar de risco operacional como } \\
\text { porcentagem do capital regulamentar mínimo total }\end{array}$ \\
\hline $\begin{array}{l}\text { 9. Outras } \\
\text { informações }\end{array}$ & $\begin{array}{l}\text { Outras informações relacionadas ao risco operacional: risco legal } \\
\text { (discussões a respeito de contingências legais, incluindo ações legais } \\
\text { pendentes, e uma discussão e estimativa de potenciais obrigações) }\end{array}$ \\
\hline
\end{tabular}

Fonte: Elaborado pelos autores com base nas recomendações do Comitê da Basiléia sobre revisão teórica e de pesquisa exploratória. 
As categorias têm como base as recomendações do Comitê da Basiléia contidas no pilar III do "Basiléia II" (BCBS, 2005) e no "Boas práticas" (BCBS, 2003b). Da revisão teórica, podem ser citadas as obras de Carvalho, Trapp e Chan (2004), Barroso, Lustosa e Moraes (2004) e Helbok e Wagner (2004). Quanto às subcategorias, a principal referência foi o já mencionado "Boas práticas" divulgado pelo Comitê da Basiléia (BCBS, 2003b), complementado pela revisão teórica já descrita para as categorias.

O Quadro 4 apresenta cada categoria associada a uma ou mais subcategorias, e não são atribuídos pesos a elas. Essa não-atribuição baseou-se na obra de Helbok e Wagner (2004), na qual não foram atribuídos pesos aos elementos de análise e em que foi possível encontrar elementos agrupados em quantidades diferentes.

A quantidade de subcategorias associada a cada categoria levou em conta o resultado da análise dos dados da pesquisa exploratória feita com profissionais, mencionada no item 3.I, em que foi constatada a aderência de dezessete subcategorias às respectivas categorias, na opinião dos respondentes. A décima oitava subcategoria, descrita no final do Quadro 4, associa-se à categoria 9, "Outras informações”. No contexto de outras informações relativas ao risco operacional, consideraram-se neste estudo as informações sobre risco legal.

Vale dizer que o risco legal foi considerado na elaboração do Quadro 4 porque está incluso no conceito de risco operacional descrito no "Basiléia II", conforme apresentado no item 2.I deste trabalho. Pesquisas realizadas pelo Comitê da Basiléia (BCBS, 200I, 2002, 2003a) e o estudo de Xavier (2003), mencionados no item 2.3 deste trabalho, mostraram discussões relativas a contingências legais como passíveis de serem verificadas empiricamente na divulgação de bancos.

\subsection{TESTES NÃO PARAMÉTRICOS}

Para dar significância estatística aos resultados encontrados nas análises dos dados secundários foram utilizados nesta pesquisa o teste U de Mann-Whitney e o teste de Wilcoxon, dois tipos de teste não paramétrico. O teste não paramétrico "não especifica condições sobre os parâmetros da população da qual se extraiu a amostra” (SIEGEL, I975). O Quadro 5 detalha os testes usados nas análises dos dados secundários. 
- ANÁLISE DO NÍVEL DE DIVULGAÇÃO DO RISCO OPERACIONAL SEGUNDO RECOMENDAÇÕES... •

\section{QUADRO 5}

TESTES USADOS NA ANÁLISE DOS DADOS SECUNDÁRIOS

\begin{tabular}{lccl}
\hline NOME DO TESTE & $\begin{array}{c}\text { TIPO DE VARIÁVEL } \\
\text { A QUE SE APLICA }\end{array}$ & $\begin{array}{c}\text { NÚMERO DE } \\
\text { AMOSTRAS }\end{array}$ & DESCRIÇÃO \\
\hline $\begin{array}{l}\text { Teste U de } \\
\text { Mann-Whitney }\end{array}$ & $\begin{array}{c}\text { Ordinal, pelo } \\
\text { menos }\end{array}$ & $\begin{array}{c}\text { Duas amostras } \\
\text { independentes }\end{array}$ & $\begin{array}{l}\text { Comprovar se dois grupos } \\
\text { independentes foram extraídos da } \\
\text { mesma população. }\end{array}$ \\
$\begin{array}{l}\text { Teste de } \\
\text { Wilcoxon }\end{array}$ & $\begin{array}{c}\text { Ordinal, pelo } \\
\text { menos }\end{array}$ & $\begin{array}{c}\text { Duas amostras } \\
\text { relacionadas }\end{array}$ & $\begin{array}{l}\text { Comprovar se duas amostras } \\
\text { proverm de uma mesma população, } \\
\text { analisando-se o sentido e valor das } \\
\text { diferenças entre os pares. }\end{array}$ \\
\hline
\end{tabular}

Fonte: Adaptado de Siegel (I975).

Finalmente, os resultados dos testes não paramétricos apresentados no item 4.3 deste estudo foram obtidos com o uso do software Statistical Package for Social Sciences (SPSS), versão I2.0.

\section{RESULTADOS}

Neste tópico, apresentam-se os resultados da divulgação por subcategoria (resultantes da análise de conteúdo), da divulgação por bancos e da aplicação dos testes não paramétricos.

\subsection{RESULTADOS DA DIVULGAÇÃO POR SUBCATEGORIA}

De acordo com a Tabela I, em cada ano a coluna "P" contém os totais de bancos que apresentaram as subcategorias presentes em seus instrumentos de divulgação. Por exemplo, para 2003 a subcategoria "Menção à integração de riscos" esteve presente na divulgação de três bancos do país, com percentual de divulgação descrito na coluna "\%P" de $\mathrm{I} 2,50 \%$. Para obter o referido percentual, foi dividida a quantidade total de bancos do país que apresentaram a subcategoria presente em 2003 (três) pela quantidade total de bancos do país avaliados (vinte e quatro). Essa forma de calcular o percentual de divulgação por subcategoria baseou-se em documentos do Comitê da Basiléia (BCBS, 200I, 2002, 2003a).

$\mathrm{Na}$ análise dos relatórios de bancos do país, observou-se a predominância da ausência de termos, exceto para a subcategoria "Outras informações relacionadas ao risco operacional: risco legal”. De acordo com a Tabela I, a subcategoria 
com maior percentual, exceto "Outras informações...", foi “Descrição dos objetivos relacionados à gestão de riscos", com 37,50\% de itens presentes em 2003 (4I,67\% em 2004). Por sua vez, "Mecanismos de incentivo à coleta de perdas operacionais", "Menção a instrumentos para transferência do risco", "Exposição ao risco operacional" e "Encargo de capital regulamentar de risco operacional" não foram mencionadas por bancos do país em 2003 e 2004 .

Para os bancos do exterior, predominou a presença de termos. Segundo a Tabela I, oito subcategorias apresentaram percentuais de I00\% tanto em 2003 quanto em 2004. Como exceção, cita-se a subcategoria "Encargo de capital regulamentar de risco operacional", a qual não foi mencionada em 2003 e 2004.

\section{TABELA I}

RESULTADOS DA DIVULGAÇÃO DO RISCO OPERACIONAL POR SUBCATEGORIA

\begin{tabular}{|c|c|c|c|c|c|c|c|c|c|}
\hline \multirow{3}{*}{$\begin{array}{l}\text { CATEGO- } \\
\text { RIAS }\end{array}$} & \multirow{3}{*}{ SUBCATEGORIAS } & \multicolumn{4}{|c|}{2003} & \multicolumn{4}{|c|}{2004} \\
\hline & & \multicolumn{2}{|r|}{ PAÍS } & \multicolumn{2}{|r|}{ EXTERIOR } & \multicolumn{2}{|r|}{ PAÍS } & \multicolumn{2}{|r|}{ EXTERIOR } \\
\hline & & $P$ & $\mathrm{P} \%$ & $P$ & $\mathrm{P} \%$ & $P$ & $\mathrm{P} \%$ & $P$ & $\mathrm{P} \%$ \\
\hline \multirow{3}{*}{$\begin{array}{l}\text { Estratégias } \\
\text { e políticas }\end{array}$} & $\begin{array}{l}\text { Descrição dos } \\
\text { objetivos relacionados } \\
\text { à gestão de riscos }\end{array}$ & 9 & $37,50 \%$ & 9 & $100,00 \%$ & 10 & $41,67 \%$ & 9 & $100,00 \%$ \\
\hline & $\begin{array}{l}\text { Menção à integração } \\
\text { de riscos }\end{array}$ & 3 & $12,50 \%$ & 8 & $88,89 \%$ & 5 & $20,83 \%$ & 8 & $88,89 \%$ \\
\hline & $\begin{array}{l}\text { Conceito de risco } \\
\text { operacional utilizado }\end{array}$ & 5 & $20,83 \%$ & 9 & $100,00 \%$ & 7 & $29,17 \%$ & 9 & $100,00 \%$ \\
\hline \multirow{4}{*}{$\begin{array}{l}\text { Estrutura } \\
\text { e organi- } \\
\text { zação da } \\
\text { função }\end{array}$} & $\begin{array}{l}\text { Envolvimento da alta } \\
\text { administração com } \\
\text { risco operacional }\end{array}$ & 6 & $25,00 \%$ & 9 & $100,00 \%$ & 9 & $37,50 \%$ & 9 & $100,00 \%$ \\
\hline & $\begin{array}{l}\text { Estrutura } \\
\text { organizacional } \\
\text { responsável pelo risco } \\
\text { operacional }\end{array}$ & 3 & $12,50 \%$ & 8 & $88,89 \%$ & 7 & $29,17 \%$ & 8 & $88,89 \%$ \\
\hline & $\begin{array}{l}\text { Segregação entre } \\
\text { gestão de riscos e área } \\
\text { de negócios do banco }\end{array}$ & 2 & $8,33 \%$ & 8 & $88,89 \%$ & 6 & $25,00 \%$ & 8 & $88,89 \%$ \\
\hline & $\begin{array}{l}\text { Segregação entre } \\
\text { auditoria interna e } \\
\text { gestão de riscos }\end{array}$ & 2 & $8,33 \%$ & 9 & $100,00 \%$ & 7 & $29,17 \%$ & 9 & $100,00 \%$ \\
\hline
\end{tabular}


- ANÁLISE DO NÍVEL DE DIVULGAÇÃO DO RISCO OPERACIONAL SEGUNDO RECOMENDAÇÕES... •

CARLOS ANDRÉ DE MELO ALVES • ANA PAULA MUSSI SZABO CHEROBIM

\section{TABELA I (CONTINUAÇÃO)}

RESULTADOS DA DIVULGAÇÃO DO RISCO

OPERACIONAL POR SUBCATEGORIA

\begin{tabular}{|c|c|c|c|c|c|c|c|c|c|}
\hline \multirow{3}{*}{$\begin{array}{l}\text { CATEGO- } \\
\text { RIAS }\end{array}$} & \multirow{3}{*}{ SUBCATEGORIAS } & \multicolumn{4}{|c|}{2003} & \multicolumn{4}{|c|}{2004} \\
\hline & & \multicolumn{2}{|r|}{ PAÍS } & \multicolumn{2}{|r|}{ EXTERIOR } & \multicolumn{2}{|r|}{ PAÍS } & \multicolumn{2}{|c|}{ EXTERIOR } \\
\hline & & $P$ & $\mathrm{P} \%$ & $P$ & $\mathrm{P} \%$ & $P$ & $\mathrm{P} \%$ & P & $\mathrm{P} \%$ \\
\hline \multirow{2}{*}{$\begin{array}{l}\text { Sistema } \\
\text { de reporte } \\
\text { interno }\end{array}$} & $\begin{array}{l}\text { Menção ao reporte da } \\
\text { área de negócios para } \\
\text { o gestor de risco }\end{array}$ & 1 & $4,17 \%$ & 8 & $88,89 \%$ & 2 & $8,33 \%$ & 9 & $100,00 \%$ \\
\hline & $\begin{array}{l}\text { Menção ao reporte do } \\
\text { gestor de risco para } \\
\text { alta administração }\end{array}$ & 3 & $12,50 \%$ & 9 & $100,00 \%$ & 2 & $8,33 \%$ & 9 & $100,00 \%$ \\
\hline \multirow{2}{*}{$\begin{array}{l}\text { Ferramen- } \\
\text { tas para } \\
\text { identifi- } \\
\text { cação e } \\
\text { avaliação } \\
\text { do risco }\end{array}$} & $\begin{array}{l}\text { Menção a ferramentas } \\
\text { para identificar } \\
\text { e avaliar o risco } \\
\text { operacional }\end{array}$ & 6 & $25,00 \%$ & 9 & $100,00 \%$ & 9 & $37,50 \%$ & 9 & $100,00 \%$ \\
\hline & $\begin{array}{l}\text { Mecanismos de } \\
\text { incentivo à coleta de } \\
\text { perdas operacionais }\end{array}$ & 0 & $0,00 \%$ & 1 & $11,11 \%$ & 0 & $0,00 \%$ & 1 & $11,11 \%$ \\
\hline \multirow{3}{*}{$\begin{array}{l}\text { Técnicas } \\
\text { para dimi- } \\
\text { nuição do } \\
\text { risco }\end{array}$} & $\begin{array}{l}\text { Menção a } \\
\text { instrumentos para } \\
\text { transferência do risco }\end{array}$ & 0 & $0,00 \%$ & 5 & $55,56 \%$ & 0 & $0,00 \%$ & 6 & $66,67 \%$ \\
\hline & $\begin{array}{l}\text { Menção a planos de } \\
\text { contingência e/ou } \\
\text { de continuidade de } \\
\text { negócios }\end{array}$ & 4 & $16,67 \%$ & 7 & $77,78 \%$ & 5 & $20,83 \%$ & 8 & $88,89 \%$ \\
\hline & $\begin{array}{l}\text { Menção a meios } \\
\text { para conscientização } \\
\text { do corpo funcional } \\
\text { quanto ao risco }\end{array}$ & 6 & $25,00 \%$ & 9 & $100,00 \%$ & 8 & $33,33 \%$ & 9 & $100,00 \%$ \\
\hline $\begin{array}{l}\text { Aborda- } \\
\text { gem para } \\
\text { avaliação } \\
\text { de capital }\end{array}$ & $\begin{array}{l}\text { Menção à abordagem } \\
\text { para avaliação de } \\
\text { capital do risco para } \\
\text { qual o banco pretende } \\
\text { se qualificar }\end{array}$ & 0 & $0,00 \%$ & 5 & $55,56 \%$ & 4 & $16,67 \%$ & 7 & $77,78 \%$ \\
\hline
\end{tabular}

(continua) 


\section{TABELA I (CONCLUSÃO)}

RESULTADOS DA DIVULGAÇÃO DO RISCO

OPERACIONAL POR SUBCATEGORIA

\begin{tabular}{|c|c|c|c|c|c|c|c|c|c|}
\hline \multirow{3}{*}{$\begin{array}{l}\text { CATEGO- } \\
\text { RIAS }\end{array}$} & \multirow{3}{*}{ SUBCATEGORIAS } & \multicolumn{4}{|c|}{2003} & \multicolumn{4}{|c|}{2004} \\
\hline & & \multicolumn{2}{|r|}{ PAÍS } & \multicolumn{2}{|c|}{ EXTERIOR } & \multicolumn{2}{|r|}{ PAÍS } & \multicolumn{2}{|r|}{ EXTERIOR } \\
\hline & & $P$ & $\mathrm{P} \%$ & P & $\mathrm{P} \%$ & $P$ & $\mathrm{P} \%$ & $P$ & $\mathrm{P} \%$ \\
\hline $\begin{array}{l}\text { Exposição } \\
\text { ao risco }\end{array}$ & $\begin{array}{l}\text { Exposição ao risco } \\
\text { operacional (valores } \\
\text { totais ou por linhas de } \\
\text { negócios) }\end{array}$ & 0 & $0,00 \%$ & 4 & $44,44 \%$ & 0 & $0,00 \%$ & 5 & $55,56 \%$ \\
\hline $\begin{array}{l}\text { Encargo } \\
\text { de capital } \\
\text { regula- } \\
\text { mentar }\end{array}$ & $\begin{array}{l}\text { Encargo de capital } \\
\text { regulamentar de risco } \\
\text { operacional como } \\
\text { porcentagem do } \\
\text { capital regulamentar } \\
\text { mínimo total }\end{array}$ & 0 & $0,00 \%$ & 0 & $0,00 \%$ & 0 & $0,00 \%$ & 0 & $0,00 \%$ \\
\hline $\begin{array}{l}\text { Outras } \\
\text { informa- } \\
\text { ções }\end{array}$ & $\begin{array}{l}\text { Outras informações } \\
\text { relacionadas ao risco } \\
\text { operacional: risco legal }\end{array}$ & 24 & $100,00 \%$ & 9 & $100,00 \%$ & 24 & $100,00 \%$ & 9 & $100,00 \%$ \\
\hline
\end{tabular}

Fonte: Dados secundários da pesquisa.

$\mathrm{P}=$ presente; País $=$ bancos do país; Exterior $=$ bancos do exterior.

De posse da quantidade de subcategorias presentes em cada ano, chegouse à Tabela 2 que apresenta o total de subcategorias divulgadas pelos bancos do país, tanto em 2003 quanto em 2004, em valores absolutos.

Conforme a Tabela 2, para os bancos do país, em 2003, verificou-se que as subcategorias relacionadas ao risco operacional estiveram presentes em instrumentos de divulgação dos bancos em 74 casos de um total de 432 possíveis (432 = I8 subcategorias x 24 bancos), o que corresponde ao percentual médio de I7,I3\%. Por sua vez, a análise de 2004 indicou que as subcategorias estiveram presentes em instrumentos de divulgação em I05 casos do mesmo total de 432 itens possíveis, o que corresponde ao percentual médio de 24,31\%. 
- ANÁLISE DO NÍVEL DE DIVULGAÇÃO DO RISCO OPERACIONAL SEGUNDO RECOMENDAÇÕES... •

\section{TABELA 2}

QUANTIDADE TOTAL DE SUBCATEGORIAS DIVULGADAS BANCOS DO PAÍS

\begin{tabular}{cccc}
\hline ANO & $\begin{array}{c}\text { TOTAL DE SUBCATEGORIAS } \\
\text { PRESENTES }\end{array}$ & $\begin{array}{c}\text { TOTAL DE SUBCATEGORIAS } \\
\text { AUSENTES }\end{array}$ & TOTAL ANALISADO \\
\hline 2003 & 74 & 358 & 432 \\
\hline 2004 & 105 & 327 & 432 \\
\hline
\end{tabular}

Fonte: Dados secundários da pesquisa.

A Tabela 3 apresenta o total de subcategorias divulgadas pelos bancos do exterior, tanto em 2003 quanto em 2004, em valores absolutos.

\section{TABELA 3}

QUANTIDADE TOTAL DE SUBCATEGORIAS DIVULGADAS BANCOS DO EXTERIOR

\begin{tabular}{cccc}
\hline ANO & $\begin{array}{c}\text { TOTAL DE SUBCATEGORIAS } \\
\text { PRESENTES }\end{array}$ & $\begin{array}{c}\text { TOTAL DE SUBCATEGORIAS } \\
\text { AUSENTES }\end{array}$ & TOTAL ANALISADO \\
\hline 2003 & 126 & 36 & 162 \\
\hline 2004 & 132 & 30 & 162 \\
\hline
\end{tabular}

Fonte: Dados secundários da pesquisa.

Referente aos bancos do exterior, segundo a Tabela 3, em 2003 verificou-se que as subcategorias relacionadas ao risco operacional estiveram presentes em instrumentos de divulgação dos bancos em I2 6 casos de um total de 162 possíveis ( $\mathrm{I} 62=\mathrm{I} 8$ subcategorias $\mathrm{x} 9$ bancos), o que corresponde ao percentual médio de $77,78 \%$. Por sua vez, a análise de 2004 indicou que as subcategorias estiveram presentes em instrumentos de divulgação em I32 casos do mesmo total de i62 possíveis, o que corresponde ao percentual médio de 8I,48\%.

Constatou-se que os bancos do exterior serviram neste estudo como uma referência internacional que permitiu a verificação de subcategorias em certos períodos que não foram encontradas nas análises dos instrumentos divulgados por bancos do país. Um exemplo foi a subcategoria "Menção à abordagem para avaliação de capital do risco para qual o banco pretende se qualificar", identificada em 2003 apenas para bancos do exterior. 


\subsection{RESULTADOS DA DIVULGAÇÃO POR BANCOS}

Apresentam-se neste item os resultados por bancos. Inicialmente, a Tabela 4 ilustra a distribuição da quantidade de subcategorias divulgadas por bancos do país em 2003 e 2004.

\section{TABELA 4}

QUANTIDADE DE SUBCATEGORIAS DIVULGADAS POR BANCOS DO PAÍS EM 2003 E 2004

\begin{tabular}{|c|c|c|c|c|c|}
\hline \multirow{2}{*}{ BANCO DO PAÍS } & \multicolumn{2}{|c|}{$\begin{array}{c}\text { QUANTIDADE DE } \\
\text { SUBCATEGORIAS DIVULGADAS }\end{array}$} & \multirow{2}{*}{ BANCO DO PAÍS } & \multicolumn{2}{|c|}{$\begin{array}{c}\text { QUANTIDADE DE } \\
\text { SUBCATEGORIAS DIVULGADAS }\end{array}$} \\
\hline & 2003 & 2004 & & 2003 & 2004 \\
\hline P04 & 11 & 13 & P15 & 1 & 2 \\
\hline P12 & 4 & 13 & P24 & 1 & 1 \\
\hline P22 & 9 & 12 & P23 & 1 & 1 \\
\hline P05 & 12 & 10 & P20 & 1 & 1 \\
\hline P21 & 5 & 10 & P19 & 1 & 1 \\
\hline P13 & 3 & 8 & P18 & 1 & 1 \\
\hline P03 & 6 & 6 & P17 & 1 & 1 \\
\hline P10 & 2 & 6 & P16 & 1 & 1 \\
\hline P08 & 4 & 5 & P11 & 1 & 1 \\
\hline P01 & 2 & 4 & P07 & 1 & 1 \\
\hline P14 & 1 & 3 & P06 & 1 & 1 \\
\hline P09 & 3 & 2 & P02 & 1 & 1 \\
\hline
\end{tabular}

Fonte: Dados secundários da pesquisa.

Segundo a Tabela 4, dos 24 bancos do país (representados de PoI a P24), dois reduziram o nível de divulgação de um ano para outro, doze mantiveram o mesmo nível, e dez divulgaram mais categorias em 2004 do que em 2003. Dentre os bancos que mantiveram a mesma quantidade de subcategorias divulgadas nos dois anos, onze divulgaram uma subcategoria apenas, a qual corresponde ao risco legal. Vale destacar que três bancos divulgaram nove ou mais subcategorias 
em 2003 (50\% ou mais das subcategorias analisadas), e este número aumentou para cinco bancos em 2004.

A análise exploratória da quantidade de subcategorias divulgadas é apresentada na Tabela 5, que indica, para cada ano, a mediana, a moda, a quantidade mínima e máxima de subcategorias divulgadas por bancos do país. A coluna "Moda" indica que a quantidade de subcategorias mais frequente em $2003 \mathrm{e}$ 2004 para os bancos foi I.

TABELA 5

ANÁLISE EXPLORATÓRIA - QUANTIDADE DE SUBCATEGORIAS DIVULGADAS POR BANCOS DO PAÍS

\begin{tabular}{ccccc}
\hline ANO & MEDIANA & MODA & QUANTIDADE MINIMA & QUANTIDADE MÁXIMA \\
\hline 2003 & 1 & 1 & 1 & 12 \\
\hline 2004 & 2 & 1 & 1 & 13 \\
\hline
\end{tabular}

Fonte: Dados secundários da pesquisa.

A Tabela 6 ilustra a distribuição da quantidade de subcategorias divulgadas em 2003 e 2004 por bancos do exterior (Eor a Eo9). Nenhum banco divulgou menos itens em 2004 do que em 2003 , quatro bancos divulgaram a mesma quantidade de itens nos dois anos, e cinco aumentaram o nível de divulgação de um ano para outro. Dentre os bancos que mantiveram a mesma quantidade de subcategorias divulgadas nos dois anos, três divulgaram $\mathrm{I} 6$ subcategorias, com o percentual de $88,89 \%$. A menor quantidade de subcategorias encontrada foi onze em $2003 \mathrm{e}$ doze em 2004, ambas atribuídas ao mesmo banco.

\section{TABELA 6}

QUANTIDADE DE SUBCATEGORIAS DIVULGADAS

POR BANCOS DO EXTERIOR EM 2003 E 2004

\begin{tabular}{ccc}
\hline & \multicolumn{2}{c}{ QUANTIDADE DE SUBCATEGORIAS DIVULGADAS } \\
\cline { 2 - 3 } BANCO DO EXTERIOR & 2003 & 2004 \\
\hline E02 & 16 & 16 \\
\hline E03 & 16 & 16 \\
\hline E06 & 16 & 16 \\
\hline
\end{tabular}




\section{TABELA 6 (CONTINUAÇÃO)}

QUANTIDADE DE SUBCATEGORIAS DIVULGADAS

POR BANCOS DO EXTERIOR EM 2003 E 2004

\begin{tabular}{ccc}
\hline & & QUANTIDADE DE SUBCATEGORIAS DIVULGADAS \\
\cline { 2 - 3 } BANCO DO EXTERIOR & 2003 & 2004 \\
\hline E07 & 14 & 15 \\
\hline E09 & 13 & 15 \\
\hline E01 & 14 & 14 \\
\hline E04 & 13 & 14 \\
\hline E05 & 13 & 14 \\
\hline E08 & 11 & 12 \\
\hline
\end{tabular}

Fonte: Dados secundários da pesquisa.

A análise exploratória da quantidade de subcategorias divulgadas por bancos do exterior está descrita na Tabela 7, a qual apresenta, para cada ano, a mediana, a moda, a quantidade mínima e máxima de subcategorias divulgadas por bancos do exterior. A coluna "Moda" indica dois valores em cada ano: I3 e I6 em 2003, e I4 e i6 em 2004.

\section{TABELA 7}

ANÁLISE EXPLORATÓRIA - QUANTIDADE DE SUBCATEGORIAS DIVULGADAS POR BANCOS DO EXTERIOR

\begin{tabular}{ccccc}
\hline ANO & MEDIANA & MODA & QUANTIDADE MÍNIMA & QUANTIDADE MÁXIMA \\
\hline 2003 & 14 & 13 e 16 & 11 & 16 \\
2004 & 15 & 14 e 16 & 12 & 16 \\
\hline
\end{tabular}

Fonte: Dados secundários da pesquisa.

\subsection{RESULTADOS DA APLICAÇÃO DOS TESTES NÃO PARAMÉTRICOS}

Com base nas informações apresentadas nas tabelas 4 e 6, foi possível aplicar os testes U de Mann-Whitney e de Wilcoxon para verificar a existência ou não 
de diferenças significativas entre a quantidade de subcategorias divulgadas pelos grupos de bancos.

Para verificar se houve diferenças significativas entre a quantidade de subcategorias divulgadas pelo grupo de bancos do país e a quantidade divulgada pelo grupo de bancos do exterior em 2003, aplicou-se o teste U de Mann-Whitney, apropriado para duas amostras independentes. A hipótese nula indicou não haver diferenças significativas entre a quantidade de subcategorias divulgadas pelos dois grupos de bancos, e a hipótese alternativa indicou haver tais diferenças. O Quadro 6 apresenta os resultados dos testes U de Mann-Whitney realizados com base em dados apresentados nas tabelas 4 e 6 .

\section{QUADRO 6}

\section{RESULTADOS DOS TESTES U DE MANN-WHITNEY}

\begin{tabular}{|c|c|c|c|c|c|}
\hline $\begin{array}{l}\text { GRUPOS DE } \\
\text { BANCOS }\end{array}$ & $\begin{array}{l}\text { NÚMERO DE } \\
\text { BANCOS }\end{array}$ & $\begin{array}{l}\text { POSTO } \\
\text { MÉDIO }\end{array}$ & TESTE & P & $\begin{array}{l}\text { RESULTADO } \\
(\text { SIG. } 0,05)\end{array}$ \\
\hline $\begin{array}{l}\text { País } 2003 \\
\text { Exterior } 2003\end{array}$ & $\begin{array}{l}24 \\
9\end{array}$ & $\begin{array}{l}12,56 \\
28,83\end{array}$ & $\begin{array}{l}U \text { de Mann-Whitney } \\
\qquad(U=1,500)\end{array}$ & 0,000 & $\begin{array}{l}\text { Com diferença } \\
\text { significativa }\end{array}$ \\
\hline $\begin{array}{l}\text { País } 2004 \\
\text { Exterior } 2004\end{array}$ & 24 & $\begin{array}{l}12,60 \\
28,72\end{array}$ & $\begin{array}{l}\text { U de Mann-Whitney } \\
\qquad(U=2,500)\end{array}$ & 0,000 & $\begin{array}{c}\text { Com diferença } \\
\text { significativa }\end{array}$ \\
\hline
\end{tabular}

Fonte: Dados secundários da pesquisa.

Conforme o Quadro 6, levando-se em conta os 24 bancos do país e os 9 do exterior em 2003, os resultados obtidos ( $\mathrm{U}=\mathrm{I}, 500 ; \mathrm{p}=0,000)$ apontaram a rejeição da hipótese nula no nível 0,05 , ou seja, a quantidade de subcategorias divulgadas pelos dois grupos de bancos em 2003 difere significativamente, no nível 0,05 . Os postos médios atribuídos a cada grupo resultantes do teste foram I2,56 para bancos do país e 28,83 para os do exterior, indicando maior divulgação para os bancos do exterior no ano.

Da mesma forma, buscou-se verificar se houve diferenças entre os níveis de divulgação do grupo de bancos do país e do grupo de bancos do exterior em 2004; nesse processo, utilizou-se novamente o teste U de Mann-Whitney. A hipótese nula indicou não haver diferenças significativas entre a quantidade de subcategorias divulgadas pelos dois grupos de bancos, no entanto a hipótese alternativa apontou a existência de diferenças.

Segundo o Quadro 6, o resultado do teste U de Mann-Whitney ( $U=2,500$; $\mathrm{p}=0,000$ ) também indicou diferenças significativas entre as quantidades de subcategorias divulgadas pelos dois grupos de bancos em 2004, no nível 0,05. 
Os postos médios atribuídos a cada grupo resultantes do teste foram I2,60 para os bancos do país e 28,72 para os do exterior, indicando maior divulgação para os bancos do exterior no ano.

Para verificar se houve diferenças significativas entre a quantidade de subcategorias divulgadas pelo grupo de bancos do país de 2003 para 2004, utilizou-se o teste de Wilcoxon, apropriado para duas amostras relacionadas. A hipótese nula indicou não haver diferenças significativas entre a quantidade de subcategorias divulgadas pelo grupo de bancos do país em 2003 e a quantidade de subcategorias divulgadas pelo mesmo grupo de bancos em 2004, no entanto a hipótese alternativa apontou a existência de diferenças.

O Quadro 7 apresenta os resultados dos testes de Wilcoxon realizados com base em dados apresentados nas tabelas 4 e 6 .

De acordo com o Quadro 7, para bancos do país, o resultado do teste de Wilcoxon $(Z=-2,485 ; p=0,013)$ indicou rejeitar a hipótese nula no nível 0,05 , ou seja, foram verificadas diferenças significativas entre a quantidade de subcategorias divulgadas em 2003 e em 2004, no nível 0,05. Como já mencionado, dois bancos do país reduziram o nível de divulgação, doze mantiveram o mesmo nível, e dez divulgaram mais categorias em 2004 do que em 2003. Os postos médios negativos e positivos resultantes do teste foram, respectivamente, 3,75 e 7,05, indicando maior divulgação em 2004.

\section{QUADRO 7}

\section{RESULTADOS DOS TESTES DE WILCOXON}

\begin{tabular}{cccccc}
\hline $\begin{array}{c}\text { GRUPOS DE } \\
\text { BANCOS }\end{array}$ & $\begin{array}{c}\text { POSTOS } \\
\text { MÉDIOS }\end{array}$ & $\begin{array}{c}\text { NÚMERO DE } \\
\text { BANCOS }\end{array}$ & TESTE & $P$ & $\begin{array}{c}\text { RESULTADO } \\
(\text { SIG. 0,05) }\end{array}$ \\
\hline País 2003 & 3,75 & 24 & $\begin{array}{c}\text { Wilcoxon } \\
(Z=-2,485)\end{array}$ & 0,013 & $\begin{array}{c}\text { Com diferença } \\
\text { significativa }\end{array}$ \\
País 2004 & 7,05 & 09 & $\begin{array}{c}\text { Wilcoxon } \\
(Z=-2,121)\end{array}$ & 0,034 & $\begin{array}{c}\text { Com diferença } \\
\text { significativa }\end{array}$ \\
\hline $\begin{array}{l}\text { Exterior 2003 } \\
\text { Exterior 2004 }\end{array}$ & 0,00 & 3,00 & 09 & $(Z=-200$ \\
\hline
\end{tabular}

Fonte: Dados secundários da pesquisa.

Da mesma maneira, para verificar se houve diferenças significativas entre a quantidade de subcategorias divulgadas pelo grupo de bancos do exterior de 2003 para 2004, foi utilizado o teste de Wilcoxon. A hipótese nula indicou não haver diferenças significativas entre a quantidade de subcategorias divulgadas pelo grupo de bancos do exterior em 2003 e a quantidade de subcategorias divulgadas pelo mesmo grupo de bancos em 2004, no entanto a hipótese alternativa apontou a existência de diferenças. 
Segundo o Quadro 7, para bancos do exterior, o resultado do teste de Wilcoxon $(\mathrm{Z}=-2$,I2I; $\mathrm{p}=0,034)$ indicou que foram verificadas diferenças significativas entre a quantidade de subcategorias divulgadas em 2003 e 2004, no nível 0,05 . Nenhum banco do exterior reduziu o nível de divulgação, quatro mantiveram o mesmo nível, e cinco divulgaram mais subcategorias em 2004 do que em 2003. Os postos médios negativos e positivos foram, respectivamente, $0,00 \mathrm{e}$ 3,00, indicando maior divulgação em 2004.

Como citado no item "Metodologia", a versão do "Basiléia II" e o Comunicado $\mathrm{n}^{\circ} \mathrm{I} 2.746$ do Banco Central do Brasil foram publicados em 2004, e analisaramse informações publicadas pelos bancos antes e depois de terem sido divulgadas tais diretrizes, verificando-se nas análises a mudança no nível de divulgação do risco operacional entre os dois anos.

Assim, as análises anteriores e os resultados dos testes indicaram que o grupo de bancos do exterior divulgou mais subcategorias do que o grupo de bancos do país, tanto em 2003 quanto em 2004. Além disso, houve aumento em 2004 na quantidade de subcategorias divulgadas pelo grupo de bancos do país e do exterior.

Finalmente, um aspecto que pode ajudar a entender as diferenças entre a quantidade de subcategorias divulgadas por bancos do exterior e do país é que foi constatado que os bancos do exterior possuíam sedes em países membros do Comitê da Basiléia. Esse fato pode ter influenciado a busca por adequação de bancos do exterior a diretrizes baseadas em recomendações do Comitê, inclusive quanto à divulgação do risco operacional.

\section{CONCLUSÃO}

O objetivo deste trabalho foi verificar os níveis de divulgação do risco operacional de 24 bancos do país e de nove do exterior, segundo recomendações do Comitê da Basiléia, nas datas-base 3I de dezembro 2003 e 3I de dezembro 2004. No período a que se refere este estudo, a divulgação desse risco foi voluntária no país.

Com base nas recomendações do Comitê contidas no pilar 3 do "Basiléia II", no "Boas práticas" e em outros estudos relacionados ao tema, abordados na revisão teórica, emergiram categorias e subcategorias de análise, as quais foram apreciadas por profissionais usuários da informação relativa ao risco operacional. As opiniões desses profissionais subsidiaram a elaboração de quadro de análise empregado para avaliar o nível de divulgação do risco operacional.

Com base nesse quadro, composto por nove categorias e I8 subcategorias, analisou-se o conteúdo de relatórios anuais e de outros instrumentos de divulga- 
ção dos bancos do país e do exterior, e foram realizados testes não paramétricos para dar significância estatística às análises dos dados secundários.

Os resultados indicaram que os níveis de divulgação do risco operacional de bancos do país e do exterior em 2003 foram, respectivamente, I7,I3\% e 77,78\%, e em 2004 os níveis foram, respectivamente, 24,31\% e 8I,48\%. Os testes não paramétricos indicaram que o grupo de bancos do exterior divulgou mais subcategorias do que o grupo de bancos do país nos dois anos e que a quantidade de subcategorias divulgadas pelos dois grupos aumentou em 2004 .

Um aspecto que pode ajudar a entender as diferenças entre a quantidade de subcategorias divulgadas por bancos do exterior e do país é que foi constatado que os bancos do exterior possuíam sedes em países membros do Comitê da Basiléia. Esse fato pode ter influenciado a busca por adequação de bancos do exterior a diretrizes baseadas em recomendações do Comitê, inclusive quanto à divulgação do risco operacional.

Quanto à divulgação por subcategoria, destacou-se que "Outras informações relacionadas ao risco operacional: risco legal" esteve presente na divulgação de todos os bancos, ao passo que a subcategoria "Encargo de capital regulamentar de risco operacional como porcentagem do capital regulamentar mínimo total" não foi mencionada.

Este estudo também buscou ampliar o conhecimento sobre a divulgação do risco operacional, apresentando quadro de análise como contribuição teórica e descrevendo o uso de testes não paramétricos na análise de dados qualitativos. Conforme citado na introdução deste trabalho, o interesse em analisar a divulgação desse risco também reside na atualidade do tema, o qual desperta atenção do público acadêmico e dos profissionais ligados ao mercado financeiro, tendo em vista a existência de diretrizes para implementação do "Basiléia II" no Brasil relacionadas ao risco operacional em instituições financeiras.

Como estudos futuros, algumas propostas de pesquisa podem ser sugeridas: verificar a influência do tamanho dos bancos sobre o nível de divulgação do risco operacional e efetuar análise comparativa entre o nível de divulgação do risco operacional e o nível de divulgação de outros riscos financeiros, como o risco de crédito e de mercado.

Finalmente, buscou-se colaborar para a aplicabilidade de recomendações do Comitê da Basiléia relativas à divulgação do risco operacional no país. Tal divulgação pode contribuir para que participantes do mercado estejam mais informados a respeito dos progressos da gestão do risco operacional em bancos, além de subsidiar as decisões de investidores e demais partes interessadas na administração de riscos de instituições financeiras. 
- ANÁLISE DO NÍVEL DE DIVULGAÇÃO DO RISCO OPERACIONAL SEGUNDO RECOMENDAÇÕES... • CARLOS ANDRÉ DE MELO ALVES • ANA PAULA MUSSI SZABO CHEROBIM

\section{REFERÊENCIAS}

BANCO CENTRAL DO BRASIL (BACEN). Comunicado $n^{\circ}$ I2.746, de 9 de dezembro de 2004 . Comunica os procedimentos para a implementação da nova estrutura de capital - Basiléia II. Disponível em: <http://www.bcb.gov.br>. Acesso em: 3 out. 2007 .

Comunicado $\mathrm{n}^{\circ} \mathrm{I}$ 6.I37, de 27 de setembro de 2007 . Comunica os procedimentos para a implementação da nova estrutura de capital - Basiléia II. Disponível em: <http://www.bcb.gov. br>. Acesso em: 3 out. $2007 \mathrm{~b}$.

. 50 maiores bancos e o consolidado do sistema financeiro nacional. Balancetes processados até 30 de março de 2005. Disponível em: <http://www.bcb.gov.br/fis/TOP50/port/ Top502004I20P.asp>. Acesso em: 3 out. 2007c.

. Relatório de estabilidade financeira. Novembro de 2004. Disponível em: <http://www. bcb.gov.br/htms/estabilidade/2004_novembro/ref2004Iocıportugues.pdf>. Acesso em: I nov. 2008a.

. Relatório de evolução do SFN de janeiro a dezembro de 2004. Disponível em: <http:// www.bcb.gov.br/htms/Deorf/ r2004I2/texto.asp?idpai=REVSFN2004I2\#Ap>. Acesso em: 2 nov. 2008b.

BARDIN, L. Análise de conteúdo. Lisboa: Edições 70, I977.

BARROSO, M. O.; LUSTOSA, P. R. B; MORAES, M. C. Disclosure de risco operacional em bancos no Brasil: evidências preliminares à luz do Novo Acordo da Basiléia. In: CONGRESSO USP DE CONTROLADORIA E CONTABILIDADE, 4., 2004, São Paulo. Anais... São Paulo: USP, 2004.

BASEL COMMITTEE ON BANKING SUPERVISION (BCBS). Public disclosures by banks: results of the I999 disclosure survey. Bank for International Settlements, April 200I. Disponível em: $<$ http://www.bis.org>. Acesso em: 3 out. 2007.

Public disclosures by banks: results of the 2000 disclosure survey. Bank for International Settlements, May 2002. Disponível em: <http://www.bis.org>. Acesso em: 3 out. 2007.

Public disclosures by banks: results of the 200I disclosure survey. Bank for International Settlements, May 2003a. Disponível em: <http://www.bis.org>. Acesso em: 3 out. 2007.

Sound practices for the management and supervision of operational risk. Bank for International Settlements, February 2003b. Disponível em: <http://www.bis.org>. Acesso em: 3 out. 2007 .

International convergence of capital measurement and capital standards - A revised framework. Bank for International Settlements, November 2005. Disponível em: <http://www. bis.org>. Acesso em: 3 out. 2007.

BRASIL. Resolução do Conselho Monetário Nacional n³.380, de 29 de junho de 2006. Dispõe sobre a implementação da estrutura de gerenciamento do risco operacional. Disponível em: $<$ http://www.bcb.gov.br>. Acesso em: 3 out. 2007.

CARVALHO, L. N.; TRAPP, A. C. G.; CHAN, B. L. Disclosure e risco operacional: uma abordagem comparativa em instituições financeiras que atuam no Brasil, na Europa e nos Estados Unidos. RAUSP, v. 39, jul./ago./set. 2004.

CROUHY, M.; GALAI, D.; MARK, R. Risk management. New York: McGraw-Hill, 200 I.

EAGOVIL, M. The intersection of corporate governance and operational risk. Bank \& Accounting Finance, Aug. 2003. 
HELBOK, G.; WAGNER, C. Corporate financial disclosure on operational risk in the banking industry. Bank Austria - Department of Operational and Group Risk Control and Vienna University of Economics and Business Administration Working Paper. This draft: September 2004. Disponível em: <http://papers.ssrn.com/sol3/papers.cfm?abstract_id=425720 >. Acesso em: I7 out. 2004 .

HENDRIKSEN, E. S.; BREDA, M. E. V. Teoria da contabilidade. 5. ed. São Paulo: Atlas, I999.

RIBEIRO, F. A. Gestão de risco operacional: indicadores-chave de risco para melhoria de processos. In: DUARTE JR., A. M.; VARGA, G. (Org.). Gestão de riscos no Brasil. Rio de Janeiro: Financial Consultoria, 2003.

SHIGVI, S. S.; DESAI, H. B. An empirical analysis of the quality of corporate financial disclosure. The Accounting Review, v. 46, n. I, p. I29-138, Jan. I97I.

SIEGEL, S. Estatística não-paramétrica (para ciências do comportamento). São Paulo: McGraw-Hill, I975.

SUNDMACHER, M. Consistency of risk reporting in financial services firms. Sydney: School of Economics and Finance, University of Western Sydney, 2006. Disponível em: <http://papers.ssrn. com/sol3/Delivery.cfm/SSRN_ID963245_code637366.pdf?abstractid=963245\&mirid=I>. Acesso em: 20 dez. 2007.

XAVIER, P. H. M. Transparência das demonstrações contábeis dos bancos no Brasil: estudo de caso sob a perspectiva do acordo "Basiléia 2". 2003. Dissertação (Mestrado em Ciências Contábeis)-Faculdade de Economia, Administração e Contabilidade, Universidade de São Paulo, São Paulo, 2003. 